\title{
Analysis of Polarimetric Multistatic Human Micro- Doppler Classification of Armed/Unarmed Personnel
}

\author{
Francesco Fioranelli, Matthew Ritchie and Hugh Griffiths \\ Department of Electronic and Electrical Engineering \\ University College London \\ Torrington Place, WC1E 7JE, London, UK
}

\begin{abstract}
Human micro-Doppler radar signatures have been investigated to classify different types of activities and to identify potential armed personnel in the context of security and surveillance applications. In this paper the use of multistatic micro-Doppler signatures to distinguish between unarmed and armed personnel moving is described. The effect of polarimetry on the classification accuracy is evaluated. Real radar data from a multistatic radar (NetRAD) has been analyzed as part of this work. Suitable features are extracted from the spectrograms generated from the data and then used as input to a classifier. The impact of polarization diversity on the classification performance is investigated, in particular the use of co-polarized or cross-polarized data or their multistatic combination.
\end{abstract}

Keywords—radar, micro-Doppler signatures, multistatic timefrequency analysis, classification

\section{INTRODUCTION}

Radar micro-Doppler is the well-known phenomenon of additional Doppler modulations caused by micro-motions on top of the main Doppler component of the main translational motion of a target $[1,2]$. For the case of human targets, microDoppler signatures are typically related to the motion of limbs and various parts of the body while a person is walking, running, or performing other movements. Human microDoppler has been investigated in applications for surveillance and security, search and rescue, and urban warfare as a means to detect, track, and classify potential human targets. Compared with other sensors and technologies, radar systems work regardless of day and night time, and they are not significantly affected by phenomena such as smoke, dust, or fog. It has been shown that micro-Doppler signatures can be analysed to characterize human gait movements along different trajectories [3], to distinguish between humans and animals [4], and potentially even to distinguish between men and women [5]. Prior micro-Doppler data from the NetRAD system has been investigated [6]. This focused on the comparison of single polarisation simulation and data results of micro-Doppler personnel walking and running. There is particular interest in using micro-Doppler data to identify people with reduced movements of their limbs, which may indicate the presence of injured people or hostages, or people who are carrying objects, which may be an indication of potential hostile activity [7]. Fully polarimetric micro-Doppler have been investigated as a promising approach to determine whether a person is armed or not [8]. The aim of this work is to explore this further and quantify possible advantages of exploiting polarization diversity in this domain as well as possible benefits of using a multistatic system to gather data.

In this paper we focus on the analysis of micro-Doppler signatures and the extraction of suitable features, then we use a classifier to distinguish between unarmed and armed personnel walking. Real co-polarized and cross-polarized data have been simultaneously recorded for the same movements and we investigate how the classification performance changes when using co-polarized or cross-polarized data, or a combination of both. In this work the walking movements have been performed on the spot, so the subjects are moving as if walking, swinging arms and raising and lowering legs and knees, but remaining in the same spatial location, without moving forward. This removes the main Doppler shift contribution from the micro-Doppler signatures and simplify the extraction of features. This also ensures that the target remains in the same range bin during the whole recording, thus avoiding a reduction of the received power caused by the target motion outside the main beams of the transmitting and receiving antennas.

The paper is organised as follows. Section II presents the experimental setup and the radar system used to record the data. Section III describes the features extracted from the micro-Doppler signatures and the classifier. Results and analysis of the classification performance are also provided in this section. Section IV finally concludes the paper.

\section{EXPERIMENTAL SETUP AND RADAR SYSTEM}

The aim of the experiment described in this work was collecting polarimetric micro-Doppler data for people walking empty-handed and walking while carrying a metallic pole which represents a rifle. The pole is approximately $1 \mathrm{~m}$ long and was held during the experiment with both hands in front of the torso, as a rifle would be. A key component of this work is the selection and extraction of suitable features from microDoppler signatures. These are used as input to a classifier to distinguish between unarmed and armed personnel. This analysis investigates the impact of polarization diversity on the performance of the classifier. 
The radar used for this experiment is the multistatic netted system NetRAD, which is a $2.4 \mathrm{GHz}, 45 \mathrm{MHz}$ bandwidth, pulsed coherent radar developed over a number of years at University College London (UCL) [9]. The experimental geometry used is shown within Fig. 1. The experiments were conducted in a large open field that represents a suitable environment with minimum ground clutter.

The monostatic transceiver node (node 3) is placed approximately $40 \mathrm{~m}$ from the other two co-located nodes (node 1 and node 2), which are the bistatic receiver-only nodes. Node 1 and 2 receive simultaneously orthogonal polarizations, namely $\mathrm{V}$-pol for node 1 and $\mathrm{H}$-pol for node 2. Node 3 receives $\mathrm{V}$-pol only, whereas the transmitted polarization was changed measurement to measurement to obtain the same amount of $\mathrm{H}$ pol and V-pol data. For the rest of the paper the polarization will be referred to by using two letters, the first indicating the transmitted polarization and the second indicating the received polarization. The transmitted/received polarization is changed by rotating of $90^{\circ}$ the antenna. The antennas used are mesh reflector antennas made by Poynting (model K-GD-03-08), with $10^{\circ}$ beam-width in both azimuth and elevation angle and approximately $24 \mathrm{dBi}$ gain. The antennas were aligned to point at the position indicated in Fig. 1 where the person performing the movement was located. This is at approximately $67 \mathrm{~m}$ from the baseline. An equal number of data has been recorded for the person facing node 1 and 2, and facing node 3 . The data presented in this paper have been collected for only one subject walking on the spot and facing these directions towards the nodes. The bistatic angle in such configuration is limited at $30^{\circ}$ as indicated.

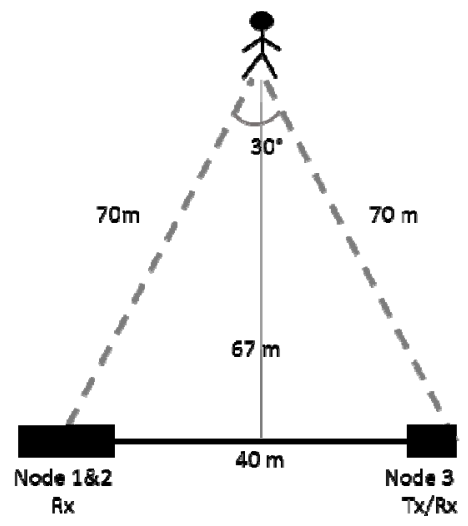

Fig. 1. Sketch of the experimental geometry

The parameters used in this experiment were a linear FM chirp pulse with $45 \mathrm{MHz}$ bandwidth, $0.6 \mu$ s pulse length, and 5 $\mathrm{kHz}$ Pulse Repetition Frequency (PRF). When used in conjunction with a high power amplifier, NetRAD can detect targets at distances up to a few kilometres [10], but in this experiment the system was operated in low power mode, with approximately $+23 \mathrm{dBm}$ transmitted power. The level of transmitted power and selected pulse length provides good detection of the subject in the range-time domain, and the PRF allows the whole human micro-Doppler signature to be recorded within the unambiguous Doppler region. Each recording consisted of 25000 pulses, equal to 5 seconds of data, which is enough to capture several periods of the average human walking gait.

\section{DATA PROCESSING}

\section{A. Feature Extraction}

Micro-Doppler signatures have been extracted from the recorded data via time-frequency analysis using Short-Time Fourier Transform (STFT). The STFTs were calculated using a 0.3 s Hamming window and 95\% overlap, followed by normalization and $40 \mathrm{~dB}$ threshold applied to the absolute value of the STFT to obtain the spectrograms of each data recording. The duration of the Hamming window was empirically chosen to provide a clean contribution of the limbs in the micro-Doppler signatures. The $40 \mathrm{~dB}$ threshold in the images of the spectrograms removes possible noise and clutter artefacts, but preserves the details of the limbs contributions to the micro-Doppler signatures.

One limitation of the walking on the spot movement is that the leg motion is not completely realistic, as the feet are not actually moving forward. However the subject is raising and lowering legs and knees, hence these body parts are still contributing to the micro-Doppler signature. Fig. 2 compares the spectrograms for a person walking on the spot facing node 3 and for actual walking towards node 3 . In both cases the data are collected at the monostatic node in $\mathrm{VV}$ polarization and the person was not carrying any object. There are some differences in the spectrograms, but these are not too significant in terms of the feature extraction technique presented in this work. The positive and negative peaks due to the limbs movement can be still appreciated when the person is walking on the spot, as well as the different intensity for limbs and main body pixels. Hence it is expected that the feature extraction and the trends in classification accuracy presented in this work should still be valid for realistic forward walking movement.
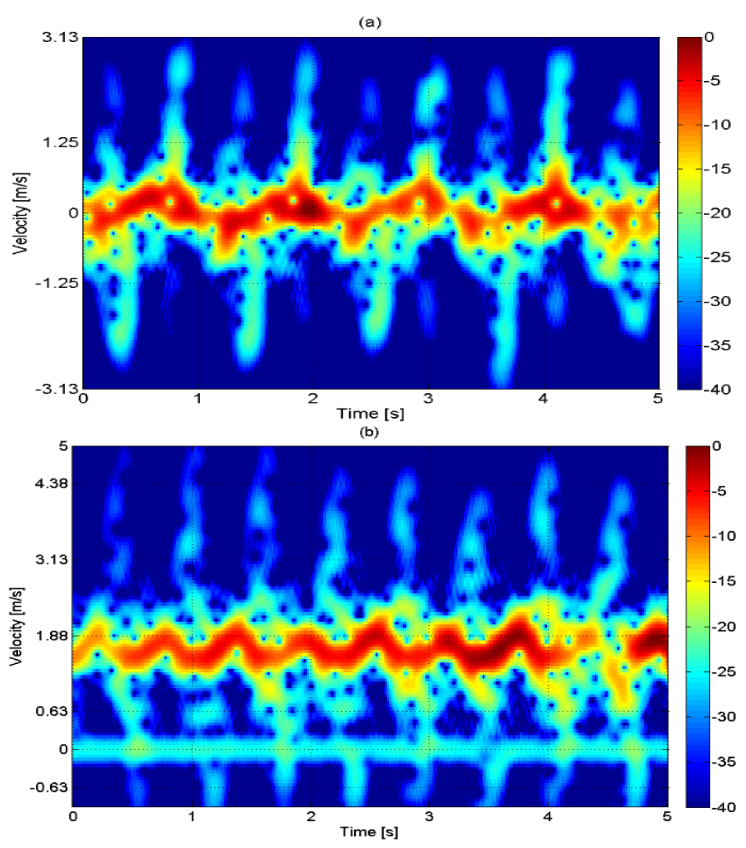

Fig. 2. Spectrograms of data recorded at mono node 3 in VV pol: subject walking on the spot facing node 3 (a) and walking towards the node (b) 
Fig. 3 shows the spectrogram of a longer recording where the person is performing three different movements, each for approximately 5 seconds. This longer recording was collected at the monostatic node with VV polarization in the same operational conditions as the 5 seconds recordings that are used for the classification. Initially the person is walking on the spot keeping hands in pockets, then normal walking with arms freely swinging, followed by walking while carrying the metallic pole representing the rifle. Around 10 seconds a short interval occurs where the bandwidth of the spectrogram is reduced and there are no positive and negative peaks. This corresponds to the moment when the person stops walking and bends down to pick up the metallic pole. This example shows empirically how the two cases of unarmed and armed personnel can be distinguished by exploiting the difference in the spectrogram, in particular in the overall Doppler bandwidth (or velocity span) of the micro-Doppler signatures caused by the different swinging movements of the arms. Intuitively, a person tends to swing less his arms while carrying objects such as a rifle because of its weight and size. In Fig. 4 it can be seen that the micro-Doppler signature spans in velocity between $\pm 3.13 \mathrm{~m} / \mathrm{s}$ (corresponding to $\pm 50 \mathrm{~Hz}$ Doppler frequencies) when the person is unarmed, but this span is reduced down to approximately $\pm 1.25 \mathrm{~m} / \mathrm{s}$ (corresponding to $\pm 20 \mathrm{~Hz}$ Doppler frequencies) when the person is carrying the simulated rifle.

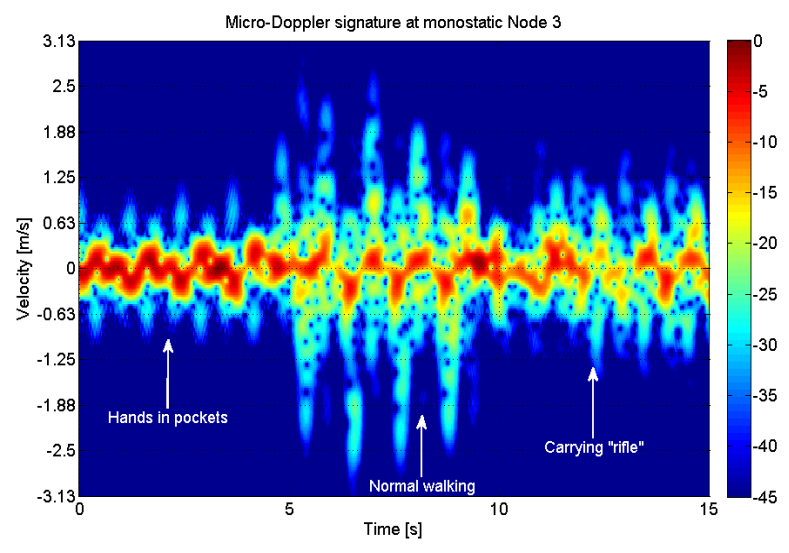

Fig. 3. Spectrogram of three consecutive activities recorded at the monostatic node in VV pol: walking with hands in pockets, walking normally, and walking while carrying a metallic pole representing a rifle.

Fig. 4 compares the spectrograms for unarmed vs armed personnel using different polarizations. These data have been recorded at the monostatic node, hence the co-polarized data on Fig. 4a and 4c are VV, and the cross-polarized data on Fig. $4 \mathrm{~b}$ and $4 \mathrm{~d}$ are HV. The figures have been normalized to their maximum, but as expected the received power for crosspolarized data is much lower than for co-polarized data. The spectrogram comparison for unarmed vs armed situation confirms what was already observed in Fig. 3, with the bandwidth of the micro-Doppler signature (i.e. the span in velocity covered by the micro-Doppler) greatly reduced in the latter case. The use of micro-Doppler to distinguish between free and confined arms swinging may be of interest, as confined arms and reduced limbs movement could be related to people carrying potentially hostile objects or to the presence of hostages or injured people. This type of analysis can exploit positive and negative micro-Doppler caused by the arms and its periodicity, and was the core contribution of these works [1113] and investigated in our previous paper [14]. These observations seem to disagree with those proposed in [15], where it is claimed that carrying objects with one or both hands does not change the micro-Doppler signature for a walking person. This may be due to the different parameters of the radar systems used to collect the data, e.g. much higher frequency $(77 \mathrm{GHz})$ and finer resolution. Nevertheless, our data show that there is a reduction of the bandwidth (or velocity span) of the micro-Doppler signature for the armed case, as in Fig. 3-4, and as reported in [11-13].
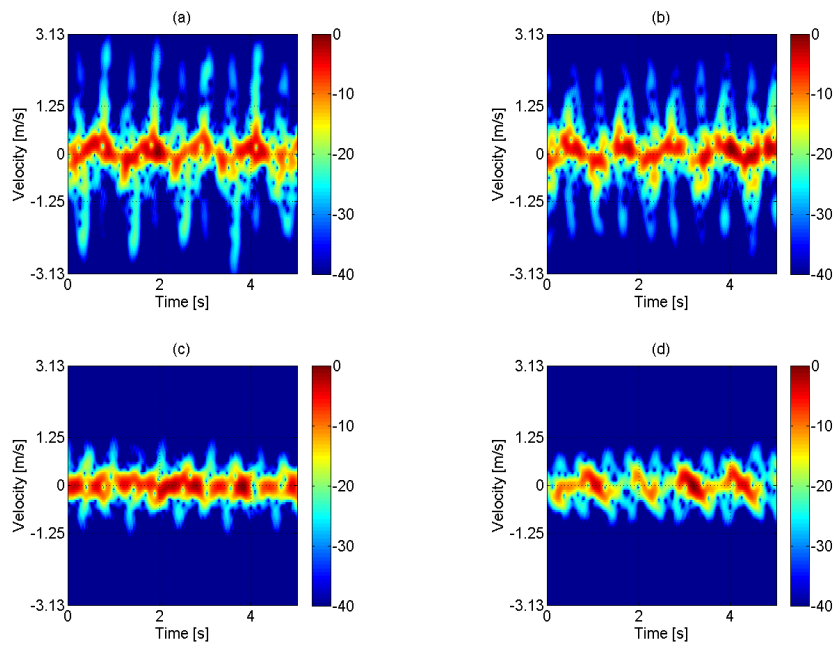

Fig. 4. Spectrograms of unarmed person walking on the spot in (a) VV pol (b) $\mathrm{HV}$ pol, and of armed person walking on the spot in (c) VV pol and (d) HV pol. These data have been collected at the monostatic node 3 .

The analysis of the STFT is followed by the extraction of features from the spectrograms to quantify the differences between the different situations of interest, in order to input such quantities to a chosen classifier. Several techniques have been proposed in the literature for this purpose. Some of them, such as Principal Component Analysis (PCA) or extraction of Cepstrum coefficients [16], are effective but transform the data into a new domain, making it more complicated to appreciate empirically the difference between the classes of interest. In this work we extracted features directly from the spectrograms trying to preserve a direct link to the kinetics of the human body. This is also a common approach in the literature for micro-Doppler analysis $[17,18]$.

Five features have been considered in this work

- Bandwidth. This is the whole range of Doppler frequencies (or corresponding velocity values) covered by the microDoppler signature and provides an indication of the amount of swinging due to the limbs movement. This can be related to the presence of an object, which may limit such swinging, as previously observed.

- Mean Period. This is the average difference in time between the micro-Doppler peaks due to the swinging of the limbs and provides an indication of the speed of the walking gait. This can provide information on the presence of objects carried by the person, as these will slow down such swinging movement because of their weight and size. 
- Doppler Offset. This is the difference between the lowest and highest Doppler frequencies recorded in the spectrograms. It would be 0 if the walking on the spot movement was perfectly symmetric, hence it can provide information on the asymmetry of the movement, which may be related to the presence of objects carried by the person.

- Radar Cross Section (RCS) Ratio between the magnitude of the micro-Doppler signature of limbs and body. This can provide information on the presence of objects, which will alter such ratio compared with walking empty-handed.

- Mean Torso Frequency. This is the average Doppler frequency of the strongest contribution in the spectrogram due to the torso of the person. In this experiment this feature is expected to be close to zero as the walking movement is on the spot, but it can still provide information on the symmetry of the movement and therefore on the presence of carried objects which may modify such symmetry (i.e. the subject may modify the micro-motions of his torso because of the encumbrance of carried objects).

Two samples of each feature have been extracted from each spectrogram, so that each feature relates to 2.5 seconds of recorded data. This has produced a database of 240 samples as a whole, considering all the three nodes. The samples are equally divided between unarmed and armed classes, as well as co-polarized and cross-polarized data, so that the database is not unbiased.

\section{B. Classification and Results}

The following step of the analysis is using feature samples as input to a classifier to identify which combinations provide the best classification performance and to investigate potential advantages in using co-polarized or cross-polarized data, or their combination. Samples of the five identified features are combined in pairs thus generating ten different combinations. Just as reference these combinations are: I bandwidth vs mean period, II bandwidth vs Doppler offset, III bandwidth vs RCS ratio, IV mean period vs Doppler offset, V mean period vs RCS ratio, VI Doppler offset vs RCS ratio, VII mean torso frequency vs bandwidth, VIII mean torso frequency vs mean period, IX mean torso frequency vs Doppler offset, and X mean torso frequency vs RCS ratio. The samples have been divided into co-polarized and cross-polarized data and input separately into two different classifiers to investigate the separate classification performance. The two classifiers used in this work are the classic Naïve Bayes classifier and the Linear Discriminant Analysis, which are described in details in the literature $[19,20]$. Both classifiers are trained with $10 \%$ of the data, which is randomly selected from the whole database, and then tested with the remaining $90 \%$ of data to obtain the confusion matrices and classification error as outputs. The process is repeated twenty times with different training datasets to validate the classifier performance in a more accurate way, and the mean value and variance of the classification error is calculated. It should be noted that two types of misclassification can happen, i.e. an unarmed case mistaken for armed (false positive) and an armed case mistaken for unarmed (false negative). In the following analysis there will be no distinction between them, as both types of mistakes are considered important to avoid. The classification error will be calculated as total number of errors for a given classifier and feature combination, divided by the whole amount of samples to be classified. It is also possible to combine in a further step the results from the classification process performed separately using co-polarized and cross-polarized data. The final decision is made considering the confidence associated to the two separate classifications and accepting the partial decision with the highest degree of confidence. This should allow to correct potential mistakes made using only one type of polarized data (co or cross) with the information provided by the other type of data. In Fig. 5 we show the bi-dimensional scatter plots for two different feature combinations, namely number I (bandwidth vs mean period) and IX (mean torso frequency vs Doppler offset). Empirically we can see that the first feature combination provides much better separation between the elements of the unarmed (in blue) and armed (in red) classes, whereas for the other combination the samples appeared overlapped in the same area. It is therefore expected that a classifier will perform much better with the first feature combination and produce a lower classification error.
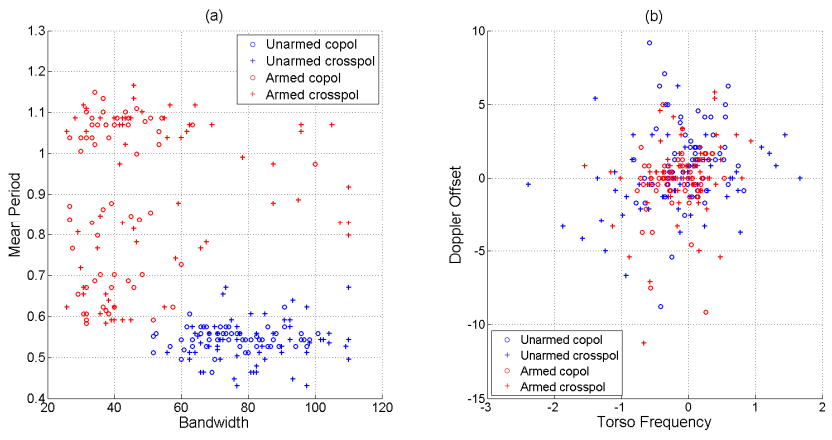

Fig. 5. Scatter plots of samples for unarmed vs armed classes for bandwidth vs mean period (a), and torso frequency vs Doppler offset (b)

Tables I and II summarize the average and variance of the classification error for all the feature combinations and classifiers considered, respectively for co-polarized and crosspolarized data. The variance of the error is consistently low, hence the validation process for the selected classifiers and the available data is considered to be accurate.

The feature combinations providing the lowest classification error and hence best performance are number I and $\mathrm{V}$ (respectively bandwidth vs mean period and mean period vs RCS ratio), with accuracy higher than $90 \%$ for both types of classifiers and for both types of polarization. Feature combination number II (bandwidth vs Doppler offset) performs as well as number I and V for the co-polarized data, but its accuracy degrades down to approximately $79 \%$ for the case of cross-polarized data. Features number IX presents the highest classification error (between $38 \%$ and $49 \%$ ), but also number VI and X do not perform very well with error around $20 \%$ for co-pol data, and even higher up to $47 \%$ for cross-pol data. This was expected from the scatter plots shown in Fig. 4, where the better (or worse) separation between elements of the different classes could be empirically seen. It is interesting to notice that 
the classification performance is not much influenced by the choice of the classifier. In other words feature combinations perform well (as number I) or bad (as number IX) for both types of classifiers, and the difference in average error is not significant. This could suggest that in developing a real system aimed at micro-Doppler classification more effort should be put in defining and extracting suitable features rather than in implementing complex and computationally heavy classifiers. However, further work with more types of classifiers and a larger database is needed to investigate this assumption.

TABLE I. MEAN AND VARIANCE OF THE CLASSIFICATION ERROR FOR DIFFERENT FEATURE COMBINATIONS AND CLASSIFIER TYPES USING ONLY COPOLARIZED DATA

\begin{tabular}{|c|c|c|c|c|}
\hline Co-pol data & \multicolumn{2}{|c|}{ Naïve Bayes } & \multicolumn{2}{c|}{ Discriminant Analysis } \\
\hline $\begin{array}{c}\text { Feature } \\
\text { Combinations }\end{array}$ & $\begin{array}{c}\text { Average } \\
\text { Error [\%] }\end{array}$ & $\begin{array}{c}\text { Error } \\
\text { Variance }\end{array}$ & $\begin{array}{c}\text { Average } \\
\text { Error [\%] }\end{array}$ & $\begin{array}{c}\text { Error } \\
\text { Variance }\end{array}$ \\
\hline I & 3.54 & 0.0011 & 4.17 & 0.0011 \\
\hline II & 9.17 & 0.0010 & 8.21 & 0.0029 \\
\hline III & 11.63 & 0.0033 & 9.79 & 0.0015 \\
\hline IV & 10.25 & 0.0034 & 15.17 & 0.0009 \\
\hline V & 4.83 & 0.0003 & 9.50 & 0.0014 \\
\hline VI & 22.29 & 0.0034 & 18.38 & 0.0015 \\
\hline VII & 10.54 & 0.0027 & 9.88 & 0.0019 \\
\hline VIII & 10.50 & 0.0067 & 15.92 & 0.0005 \\
\hline IX & 41.38 & 0.0032 & 38.33 & 0.0058 \\
\hline X & 24.92 & 0.0067 & 21.00 & 0.0018 \\
\hline
\end{tabular}

TABLE II. MEAN AND VARIANCE OF THE CLASSIFICATION ERROR FOR DIFFERENT FEATURE COMBINATIONS AND CLASSIFIER TYPES USING ONLY CROSS-POLARIZED DATA

\begin{tabular}{|c|c|c|c|c|}
\hline Cross-pol data & \multicolumn{2}{|c|}{ Naïve Bayes } & \multicolumn{2}{c|}{ Discriminant Analysis } \\
\hline $\begin{array}{c}\text { Feature } \\
\text { Combinations }\end{array}$ & $\begin{array}{c}\text { Average } \\
\text { Error [\%] }\end{array}$ & $\begin{array}{c}\text { Error } \\
\text { Variance }\end{array}$ & $\begin{array}{c}\text { Average } \\
\text { Error [\%] }\end{array}$ & $\begin{array}{c}\text { Error } \\
\text { Variance }\end{array}$ \\
\hline I & 4.67 & 0.0019 & 7.38 & 0.0034 \\
\hline II & 21.33 & 0.0043 & 21.08 & 0.0030 \\
\hline III & 20.04 & 0.0018 & 17.83 & 0.0050 \\
\hline IV & 14.13 & 0.0007 & 12.54 & 0.0023 \\
\hline V & 9.67 & 0.0011 & 7.13 & 0.0037 \\
\hline VI & 31.21 & 0.0044 & 42.54 & 0.0037 \\
\hline VII & 23.54 & 0.0057 & 24.38 & 0.0024 \\
\hline VIII & 13.17 & 0.0009 & 13.04 & 0.0022 \\
\hline IX & 46.17 & 0.0026 & 49.58 & 0.0016 \\
\hline X & 30.63 & 0.0007 & 47.46 & 0.0030 \\
\hline
\end{tabular}

In table III the results of average classification error and variance obtained from the combination of co-polarized and cross-polarized data are shown. Feature combinations number I and $\mathrm{V}$ are still those providing the best performance, whereas combination number IX has the highest classification error. In case of feature combinations I and V, the benefit of combining information from co-polarized and cross-polarized data can be seen in the overall lower classification error compared with the previous two tables. Where the classification accuracy is now higher than $95 \%$ (error lower than 5\%). The same phenomenon of decreased error can be also seen for feature combinations number IV and VIII, but this does not happen for the other feature combinations for which the error remains similar or even slightly increases.
TABLE III. MEAN AND VARIANCE OF THE CLASSIFICATION ERROR FOR DIFFERENT FEATURE COMBINATIONS AND CLASSIFIER TYPES COMBINING COPOLARIZED AND CROSS-POLARIZED DATA

\begin{tabular}{|c|c|c|c|c|}
\hline Combined data & \multicolumn{2}{|c|}{ Naïve Bayes } & \multicolumn{2}{c|}{ Discriminant Analysis } \\
\hline $\begin{array}{c}\text { Feature } \\
\text { Combinations }\end{array}$ & $\begin{array}{c}\text { Average } \\
\text { Error [\%] }\end{array}$ & $\begin{array}{c}\text { Error } \\
\text { Variance }\end{array}$ & $\begin{array}{c}\text { Average } \\
\text { Error [\%] }\end{array}$ & $\begin{array}{c}\text { Error } \\
\text { Variance }\end{array}$ \\
\hline I & 1.75 & 0.0009 & 3.71 & 0.0008 \\
\hline II & 10.88 & 0.0038 & 8.79 & 0.0017 \\
\hline III & 12.67 & 0.0007 & 9.67 & 0.0033 \\
\hline IV & 7.75 & 0.0008 & 6.71 & 0.0032 \\
\hline V & 3.83 & 0.0018 & 5.00 & 0.0022 \\
\hline VI & 22.29 & 0.0015 & 20.58 & 0.0035 \\
\hline VII & 10.67 & 0.0016 & 9.75 & 0.0013 \\
\hline VIII & 7.17 & 0.0012 & 7.67 & 0.0057 \\
\hline IX & 41.38 & 0.0051 & 39.33 & 0.0015 \\
\hline X & 22.63 & 0.0031 & 24.21 & 0.0057 \\
\hline
\end{tabular}

\section{CONCLUSIONS}

In this paper the use of micro-Doppler signatures to distinguish between armed vs unarmed personnel has been investigated with polarimetric experimental data collected by the UCL NetRAD system. Five features have been extracted from the spectrograms of the data and used in pairs as input to two different classifiers, Naïve Bayes and Linear Discriminant Analysis. Suitable features which provide classification accuracy up to $90-95 \%$ have been identified, namely bandwidth vs mean period and mean period vs RCS body-limb ratio. These features appear to provide the best classification results regardless of the classifier type and polarization.

The effect of polarization diversity has also been investigated, performing separate classification and decision on co-polarized and cross-polarized data, and then combining information from both types of data on the basis of the highest classification confidence. The overall result of combining information from different polarizations has shown positive improvements in classification accuracy, although further work and data are necessary to fully clarify potential advantages of the multi-polarization approach. One issue to investigate is whether a different feature extraction process is needed for copolarized and cross-polarized data to optimize the amount of information extracted.

Future work will include collecting additional data with different subjects to give stronger statistical confidence to the preliminary results presented in this work. Different geometries with different bistatic angles and different aspect angles will be also analysed to investigate changes in classification accuracy as a combined function of these variables and polarization diversity. Different ways of combining information from different multistatic radar nodes will also be explored, in order to maximise the classification accuracy. This will extend the results presented in [14]. Furthermore, other types of classifiers such as Nearest Neighbour and Support Vector Machine may be tested with the data, as well as different approaches to extract features such as PCA or Cepstrum coefficients.

\section{ACKNOWLEDGMENT}

The authors would like to thank all the members of the UCL Radar Group who helped in setting up and conducting the field trials. This work has been funded by the IET A F Harvey Prize, awarded to Hugh Griffiths (2013). 


\section{REFERENCES}

[1] V. C. Chen, L. Fayin, S. S. Ho and H. Wechsler, "Micro-Doppler effect in radar: phenomenon, model, and simulation study", IEEE Trans. On Aerospace and Electronic Systems, vol. 42, pp. 2-21, 2006.

[2] V. C. Chen, D. Tahmoush and W. L. Miceli, Radar Micro-Doppler Signatures: Processing and Applications, Institution of Engineering and Technology, 2014.

[3] D. Tahmoush and J. Silvious, "Radar micro-Doppler for long range front-view gait recognition", IEEE $3^{\text {rd }}$ International Conference on Biometrics: Theory, Applications, and Systems, pp 1-6, Washington DC, USA, 2009.

[4] D. Tahmoush and J. Silvious, "Remote detection of humans and animals", IEEE Applied Imagery Pattern Recognition Workshop, pp. 18, Washington DC, USA, 2009.

[5] D. Tahmoush and J. Silvious, "Radar microDoppler for security applications: Modeling men versus women", IEEE Antennas and Propagation Society International Symposium, pp. 1-4, North Charleston, South Carolina, 2009.

[6] Smith, G.E., Woodbridge, K., Baker, C.J., and Griffiths, H.D., 'Multistatic Micro-Doppler Radar Signatures of Personnel Targets', IET Signal Processing, 4, (3), pp. 224-233, 2010.

[7] Tivive, F.H.C., Bouzerdoum, A., and Amin, M.G., 'Automatic Human Motion Classification from Doppler Spectrograms', CIP 2nd International Workshop on Cognitive Information Processing, Jun. 2010, Elba Island, Italy.

[8] Tahmoush, D. and Silvious, J., 'Radar Polarimetry for Security Applications', EuRAD European Radar Conference, Sep. 2010, Paris, France.

[9] Derham, T.E., Doughty, S., Woodbridge, K., and Baker, C.J., 'Design and Evaluation of a Low-Cost Multistatic Netted Radar System', IET Radar, Sonar \& Navigation, vol. 1, no. 5, pp. 362-368, 2007.

[10] Al-Ashwal, W.A., Baker, C.J., Balleri, A., Griffiths, H.D., Harmanny, R., Inggs, M., Miceli, W.J., Ritchie, M., Sandenbergh, J.S., Stove, A., Tough, R.J.A., Ward, K.D., Watts, S., and Woodbridge, K., 'Statistical Analysis of Simultaneous Monostatic and Bistatic Sea Clutter at Low Grazing Angles', Electronics Letters, vol. 47, no. 10, 2011.
[11] Mobasseri, B.G. and Amin, M.G., 'A Time-Frequency Classifier for Human Gait Recognition', Proceedings of Optics and Photonics in Global Homeland Security $V$ and Biometric Technology for Human Identification, Apr. 2009, Orlando, USA.

[12] Orović, I., Stanković, S., and Amin, M., 'A New Approach for Classification of Human Gait Based on Time-Frequency Feature Representations', Signal Processing, 91, (6), pp. 1448-1456, 2011.

[13] Tivive, F., Bouzerdoum, S., and Amin, M., A Human Gait Classification Method Based on Radar Doppler Spectrograms', EURASIP Journal on Advances in Signal Processing, Vol. 2010, Art. ID 389716.

[14] Fioranelli, F., Ritchie, M., and Griffiths, H., 'Multistatic Human MicroDoppler Classification of Armed/Unarmed Personnel', IET Radar, Sonar \& Navigation, Accepted for publication in January 2015.

[15] Bjorklund, S., Petersson, H., Nezirovic, A., Guldogan, M.B., and Gustafsson, F., 'Millimeter-Wave Radar Micro-Doppler Signatures of Human Motion', Proceedings of IRS International Radar Symposium, Sep. 2011, Leipzig, Germany.

[16] Balleri, A., Chetty, K., and Woodbridge, K., 'Classification of Personnel Targets by Acoustic Micro-Doppler Signatures', IET Radar, Sonar \& Navigation, 5, (9), pp. 943-951, 2011.

[17] Youngwook, K. and Hao, L., 'Human Activity Classification Based on Micro-Doppler Signatures Using a Support Vector Machine', IEEE Trans. On Geoscience and Remote Sensing, 47, (5), pp. 1328-1337, 2009.

[18] Youngwook, K. and Hao, L., 'Human Activity Classification Based on Micro-Doppler Signatures Using an Artificial Neural Network', $A P-S$ 2008 IEEE International Symposium on Antennas and Propagation, Jul. 2008, San Diego, USA.

[19] Fisher, R.A., 'The Use of Multiple Measurements in Taxonomic Problems', Annals of Eugenics, 1936, 7, (2), pp. 179-188.

[20] Hastie, T., Tibshirani, R., and Friedman, J., The Elements of Statistical Learning: Data Mining, Inference, and Prediction, Second Edition, (Springer, 2009). 